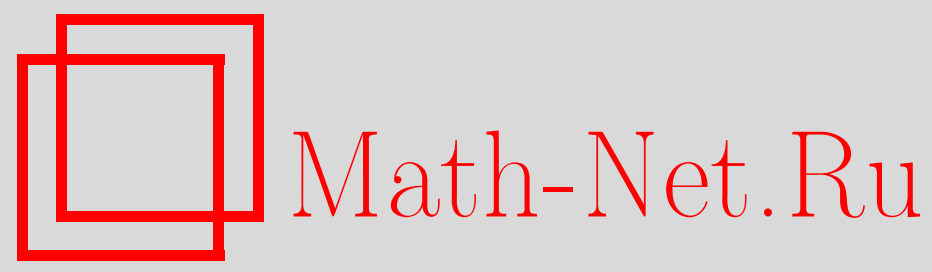

А. П. Розовская, Д. А. Шабанов, О правильных раскрасках гиперграфов в предписанные цвета, Дискрет. матем., 2010, том 22, выпуск 3, 94-109

DOI: https://doi.org/10.4213/dm1110

Использование Общероссийского математического портала Math-Net.Ru подразумевает, что вы прочитали и согласны с пользовательским соглашением http://www . mathnet.ru/rus/agreement

Параметры загрузки:

IP: 54.81 .137 .203

26 апреля 2023 г., $12: 37: 48$ 


\title{
О правильных раскрасках гиперграфов в предписанные цвета
}

\author{
() 2010 г. А. П. Розовская, Д. А. Шабанов
}

\begin{abstract}
Рассматривается обобщение классической задачи Эрдеша-Хайнала в теории гиперграфов на случай предписанных раскрасок. Изучается величина $m_{p r}(n, r)$, равная минимальному числу ребер гиперграфа в классе $n$-равномерных гиперграфов, предписанное хроматическое число которых больше $r$. Получена нижняя оценка данной величины, которая улучшает ранее известные результаты для $r \geqslant 3$. Кроме того, указано достаточное условие существования предписанной $r$-раскрашиваемости $n$-равномерного гиперграфа в терминах ограничений на пересечение ребер. В качестве следствия получена новая нижняя оценка величины $m_{p r}^{*}(n, r)$, равной минимальному числу ребер гиперграфа в классе $n$-равномерных простых гиперграфов (в которых любые два ребра имеют не более одной общей вершины), предписанное хроматическое число которых больше $r$.

Работа выполнена при поддержке Российского фонда фундаментальных исследований, грант 06-01-00383, и программы Президента Российской Федерации поддержки ведущих научных школ, грант НШ 691.2008.1.
\end{abstract}

\section{1. Введение и история классической задачи}

Данная работа посвящена обобщению классической задачи Эрдеша-Хайнала в теории гиперграфов. Напомним, что гиперграфом называется пара множеств $H=(V, E)$, где $V$ есть некоторое конечное множество, называемое множеством вершин гиперграфа, а $E$ есть совокупность подмножеств множества $V$, эти подмножества называются ребрами гиперграфа. Гиперграф является $n$-равномерным, если каждое его ребро содержит ровно $n$ вершин. Хроматическим числом гиперграфа $H$ называется минимальное число цветов, в которые можно так покрасить множество $V$ вершин гиперграфа, что все ребра из $E$ будут неодноцветными. Мы будем обозначать хроматическое число гиперграфа $H$ через $\chi(H)$.

В 1961 г. П. Эрдеш и А. Хайнал (см. [1, 2]) поставили задачу об отыскании величины $m(n, r)$, равной минимально возможному числу ребер в $n$-равномерном гиперграфе с хроматическим числом, большим $r$. Позднее Эрдеш (см. [3, 4]) доказал первые нетривиальные оценки величины $m(n, 2)$. С помощью простого вероятностного метода он показал, что при $n \rightarrow \infty$

$$
2^{n-1} \leqslant m(n, 2) \leqslant(1+o(1)) \frac{e \ln 2}{4} n^{2} 2^{n} .
$$

Похожие оценки верны и для произвольного $r$. А именно, для любых $r \geqslant 2$ и $n \rightarrow \infty$ 
выполнены (см. [5]) неравенства

$$
r^{n-1} \leqslant m(n, r) \leqslant \frac{e \ln r}{2} n^{2} r^{n}(1+O(1 / n)) .
$$

Нижняя оценка величины $m(n, 2)$ была последовательно улучшена Шмидтом (см. [6]) и Беком (см. [7, 8]). Дж. Спенсер (см. [9]) предложил более простой вариант доказательства оценки Бека, основанный на применении рандомизированного алгоритма перекраски вершин гиперграфа. Наконец, в 2000 г. Дж. Радхакришнаном и А. Сринивазаном был получен (см. [10]) наилучший на сегодняшний день результат для случая двух цветов. Они доказали, что

$$
m(n, 2) \geqslant 0,2\left(\frac{n}{\ln n}\right)^{1 / 2} 2^{n-1} .
$$

Исследования поведения величины $m(n, r)$ при большом $r$ были проведены Н. Алоном (см. [11]). Он показал, что для произвольных $n$ и $r$ выполняется неравенство

$$
m(n, r)>(n-1)\lceil r / n\rceil\lfloor(n-1) r / n\rfloor^{n-1} .
$$

Сильная асимптотическая нижняя оценка была получена А. В. Косточкой (см. [12]), использовавшим многоступенчатый аналог рандомизированного алгоритма, предложенного Радхакришнаном и Сринивазаном. Согласно данному результату, в случае, когда $r$ не превосходит $\sqrt{(1 / 8) \ln (\ln n / 2)}$, величина $m(n, r)$ оценивается снизу следующим образом:

$$
m(n, r) \geqslant r^{n}(n / \ln n)^{m /(m+1)} e^{-4 r^{2}},
$$

где $m=\left\lfloor\log _{2} r\right\rfloor$.

\section{2. Обобщение задачи для предписанных раскрасок}

Сначала напомним ряд определений. Пусть $H=(V, E)-$ некоторый гиперграф и задано конечное множество $C$, элементы которого мы будем называть цветами. Вершинным предписанием $A$ называется любое отображение, которое каждой вершине $v \in V$ ставит в соответствие некоторое подмножество $A(v) \subseteq C$. Если $|A(v)|=r$ для любой вершины $v \in V$, то будем говорить, что мощность предписания равна $r$.

Раскраской $f$ вершин гиперграфа $H$, соответствующей предписанию $A$, называется такое однозначное отображение $f: V \rightarrow C$, что $f(v) \in A(v)$ для любого $v \in V$. При этом будем говорить, что каждая вершина $v$ окрашена в цвет $f(v)$. Раскраска называется правильной для гиперграфа $H$, если в этой раскраске все ребра гиперграфа неодноцветны. Гиперграф называется предписанно $r$-раскрашиваемым, если для любого множества цветов $C$ и любого вершинного предписания $A$ мощности $r$ найдется правильная раскраска, соответствующая данному предписанию. Предписанным хроматическим числом гиперграфа $H$ называется такое минимальное натуральное число $r$, что $H$ является предписанно $r$-раскрашиваемым.

Изучение предписанного хроматического числа было инициировано работами В. Г. Визинга [13], а также П. Эрдеша, А. Л. Рубина и Х. Тейлора [14]. Для предписанного хроматического числа гиперграфа $H$ мы будем использовать обозначение $\chi_{p r}(H)$.

В работе [12] (см. также [5]) А. В. Косточка сформулировал обобщение экстремальной задачи Эрдеша-Хайнала для предписанных раскрасок. Он предложил найти величину 
$m_{p r}(n, r)$, равную минимально возможному числу ребер гиперграфа в классе $n$-равномерных гиперграфов, предписанное хроматическое число которых превышает $r$. В силу того, что для любого гиперграфа $H$ выполнено неравенство

$$
\chi(H) \leqslant \chi_{p r}(H),
$$

соотношение между величинами $m(n, r)$ и $m_{p r}(n, r)$ будет обратным, то есть

$$
m(n, r) \geqslant m_{p r}(n, r) .
$$

Несложно проверить, что для $m_{p r}(n, r)$ выполняются те же оценки, что и для величины $m(n, r)$ в (1), а именно,

$$
r^{n-1} \leqslant m_{p r}(n, r) \leqslant \frac{e \ln r}{2} n^{2} r^{n}\left(1+O\left(\frac{1}{n}\right)\right) .
$$

Верхняя оценка, конечно, сразу следует из (5), а нижнюю можно доказать, используя, как и раньше, простые вероятностные соображения.

А. В. Косточка отметил (см. [12]), что доказательство оценки Радхакришнана-Сринивазана (2) проходит и в случае предписанных раскрасок, а вот методы, с помощью которых были обоснованы оценки (3) и (4), не позволяют получить оценки величины $m_{p r}(n, r)$. Теорема 1 дает нижнюю оценку для $m_{p r}(n, r)$ в случае произвольного $r$.

Теорема 1. Для всех натуральных $n \geqslant 2, r \geqslant 2$ выполняется неравенство

$$
m_{p r}(n, r) \geqslant(\sqrt{3}-1)(n / \ln n)^{1 / 2} r^{n-1} .
$$

Ясно, что (7) улучшает нижнюю оценку из (6) для всех значений параметров $n$ и $r$. Из соотношения (5) мы получаем простое следствие из теоремы 1.

Следствие 1. Для всех натуральных $n \geqslant 2, r \geqslant 2$ выполняется неравенство

$$
m(n, r)(\sqrt{3}-1)(n / \ln n)^{1 / 2} r^{n-1} .
$$

Проведем небольшое сравнение оценки (8) с известными ранее оценками (1)-(4).

1. При всех значениях $n$ и $r$ оценка (8) улучшает нижнюю оценку из (1).

2. При $r=2$ оценка (8) имеет тот же порядок роста, что и оценка РадхакришнанаСринивазана (2). Различие констант не является принципиальным (см. замечание 1 ниже).

3. Результат Алона (3) лучше (8) только для больших значений $r$. Правая часть неравенства (3) при фиксированном $n$ и растущем $r$ имеет порядок роста $r^{n}$, что существенно превышает (8). Но, как несложно проверить, при $r$, удовлетворяющем условию

$$
r \leqslant \frac{2(n-1)}{\ln (n \ln n)-2 \ln (\sqrt{3}-1)} \sim \frac{2 n}{\ln n},
$$

оценка (8) становится лучше оценки (3), правая часть которой имеет при данных ограничениях вид $(n-1)(r-1)^{n-1}$.

4. Оценка (4) А. В. Косточки лучше (8), но она выполняется лишь для значений $r=O(\sqrt{\ln \ln n})$, в то время как (8) верна для всех возможных значений $n$ и $r$. 
Метод, с помощью которого проводится доказательство теоремы 1, построен на обобщении идей, лежащих в основе рандомизированных алгоритмов двухцветной раскраски, разработанных Спенсером и Радхакришнаном со Сринивазаном.

Замечание 1. Отметим, что, вообще говоря, константа $\sqrt{3}-1$ в оценке (7) не является оптимальной. Ее можно заменить на большую величину при условии, что параметры $n$ и $r$ достаточно велики. Однако, нам более интересен порядок роста величин, нежели точное вычисление констант при растущих множителях.

Теорему 1 мы докажем в разделе 4, а в следующем разделе сформулируем некоторые важные следствия из нее.

\section{3. Следствия из основного результата}

В данном разделе мы приведем следствия теоремы 1.

\section{1. Достаточное условие $r$-раскрашиваемости гиперграфов}

В известной совместной работе П. Эрдеша и Л. Ловаса [15] было получено достаточное условие существования правильной $r$-цветной раскраски для равномерных гиперграфов в терминах ограничений на число попарных пересечений ребер. Эрдеш и Ловас доказали, что если каждое ребро $n$-равномерного гиперграфа $H$ пересекается не более чем с $r^{n-1} / 4$ другими ребрами, то $\chi(H) \leqslant r$. Данный результат был улучшен Дж. Радхакришнаном и А. Сринивазаном (см. [10]) в случае $r=2$. Для больших значений $n$ они показали, что если каждое ребро $n$-равномерного гиперграфа пересекается не более чем с $0,17 \sqrt{n / \ln n} 2^{n}$ другими ребрами, то хроматическое число такого гиперграфа не превосходит двух. С помощью построенной в доказательстве теоремы 1 случайной раскраски (см. раздел 4) можно получить подобный результат для произвольного $r$. Справедлива следующая теорема.

Теорема 2. Пусть $n \geqslant 2, r \geqslant 2-$ натуральнье числа,

$$
d=\frac{\sqrt{6}-2}{4}\left(\frac{n}{\ln n}\right)^{1 / 2} r^{n-1} .
$$

Если $H-n$-равномерный гиперграф, каждое ребро которого пересекается не более чем c d другими ребрами гиперграфа, то

$$
\chi_{p r}(H) \leqslant r .
$$

При всех $r$ и $n \geqslant 13$ теорема 2 улучшает результат Эрдеша-Ловаса. Доказательство теоремы 2 мы приведем в разделе 6 .

\section{2. Простые гиперграфы}

В задачах о минимальном числе ребер гиперграфа, принадлежащего определенному классу, можно наложить дополнительные ограничения на этот класс помимо $n$-равномерности и значения хроматического числа, которые имели место в задаче об отыскании величины $m(n, r)$. Одним из классических подобных ограничений является требование простоты гиперграфа. Гиперграф называется простым, если любые два ребра этого гиперграфа

4 Дискретная математика, т.22 №3 
имеют не более одной общей вершины. По аналогии с $m(n, r)$ обозначим через $m^{*}(n, r)$ минимальное число ребер гиперграфа в классе простых $n$-равномерных гиперграфов, хроматическое число которых превышает $r$.

Задача о нахождении величины $m^{*}(n, r)$ была поставлена в работе П. Эрдеша и Л. Ловаса [15]. В ней же были получены первые оценки для $m^{*}(n, r)$. Эрдеш и Ловас показали, что для любых $n \geqslant 2, r \geqslant 2$ выполняются неравенства

$$
\frac{r^{2(n-2)}}{16 n(n-1)^{2}} \leqslant m^{*}(n, r) \leqslant 1600 n^{4} r^{2(n+1)} .
$$

Верхняя оценка по-прежнему является наилучшей, если $n$ велико по сравнению с $r$. Нижняя оценка может быть улучшена в $n / 2$ раз за счет некоторых простых соображений о сумме степеней вершин простого гиперграфа (подробнее см. [5]). Таким образом, фактически Эрдешем и Ловасом было доказано, что

$$
m^{*}(n, r) \geqslant \frac{r^{2(n-2)}}{32(n-1)^{2}} .
$$

В случае $r=2$ нижняя оценка была дополнительно улучшена. 3. Сабо (см. [16]) доказал, что для любого $\varepsilon>0$ существует такое натуральное $n_{0}=n_{0}(\varepsilon)$, что при $n \geqslant n_{0}$ любой простой $n$-равномерный гиперграф с хроматическим числом 3 содержит вершину степени, не меньшей, чем $n^{\varepsilon} 2^{n}$. Из этого факта, следуя рассуждениям Эрдеша и Ловаса из [15], несложно получить следующую оценку величины $m^{*}(n, 2)$ : для любого $\varepsilon>0$ существует такое натуральное $n_{0}=n_{0}(\varepsilon)$, что при $n \geqslant n_{0}$ выполняется неравенство

$$
m^{*}(n, 2) \geqslant \frac{4^{n-1}}{2 n^{2 \varepsilon}} .
$$

Также величина $m^{*}(n, r)$ для любого $n$ и бесконечно многих $r$ оценивается сверху следующим выражением:

$$
m^{*}(n, r) \leqslant c 4^{n} n^{2} r^{2 n-2} \ln ^{2} r, \quad c>0 .
$$

Этот результат был получен в [17]. В случае, когда $r$ велико по сравнению с $n$, данная оценка улучшает результат Эрдеша-Ловаса (10).

По аналогии с $m^{*}(n, r)$ введем величину $m_{p r}^{*}(n, r)$ как минимальное число ребер гиперграфа в классе простых $n$-равномерных гиперграфов, предписанное хроматическое число которых превышает $r$. Теорема 3 дает нижнюю оценку для $m_{p r}^{*}(n, r)$.

Теорема 3. Для всех натуральных $n \geqslant 2, r \geqslant 2$ выполняется неравенство

$$
m_{p r}^{*}(n, r) \geqslant \frac{(\sqrt{6}-2)^{2}}{32} \frac{r^{2 n-4}}{n \ln n} .
$$

Следствие 2. Для всех натуральных $n \geqslant 2, r \geqslant 2$ выполняется неравенство

$$
m^{*}(n, r) \geqslant \frac{(\sqrt{6}-2)^{2}}{32} \frac{r^{2 n-4}}{n \ln n} .
$$

Утверждение следствия 2 легко вытекает из теоремы 2 в силу справедливости соотношения

$$
m_{p r}^{*}(n, r) \leqslant m^{*}(n, r)
$$

которое аналогично (5). Отметим, что полученная нами оценка (14) лучше (11) при $n \geqslant 16$, но для $r=2$ асимптотически хуже оценки (12). 
Замечание 2. Константы в оценках (9) и (13) можно улучшить при больших значениях параметров $n$ и $r$. Однако, как уже отмечалось в замечании 1 , нас интересует порядок роста величин, а не точные значения констант при растущих множителях.

Доказательство теоремы 3 будет приведено в разделе 7.

\section{4. Доказательство теоремы 1}

Доказательство теоремы 1 основано на применении метода случайной раскраски вершин гиперграфа. Для обоснования неравенства (7) достаточно показать, что для любого $n$-равномерного гиперграфа $H=(V, E)$, имеющего менее $(\sqrt{3}-1) \sqrt{n / \ln n} r^{n-1}$ ребер, справедливо неравенство

$$
\chi_{p r}(H) \leqslant r .
$$

Итак, пусть фиксирован $n$-равномерный гиперграф $H=(V, E)$ с $m$ ребрами (то есть $|E|=m)$. Пусть также задано конечное множество цветов $C$ и вершинное предписание $A$ мощности $r$, сопоставляющее каждой вершине $v \in V$ множество $A(v) \subset C$. Без ограничения общности можно считать, что $V=\{1,2, \ldots, w\}$, а $C \subset \mathbf{N}$. Мы построим некоторую случайную раскраску $\zeta$, соответствующую данному предписанию, и оценим вероятность того, что эта раскраска является правильной для $H$.

Пусть на некотором вероятностном пространстве задан следующий набор независимых в совокупности случайных функций:

(1) случайные величины $\xi_{1}, \ldots, \xi_{w}$ со следующим распределением: $\xi_{v}$ принимает все значения из $A(v)$ с равной вероятностью $1 / r$;

(2) случайные величины $\eta_{1}, \ldots, \eta_{w}$ со следующим распределением: $\eta_{v}$ принимает все значения из $A(v)$ с одной и той же вероятностью $p \in[0,1 / r]$ и значение 0 с вероятностью $1-r p$;

(3) $\sigma=\left(\sigma_{1}, \ldots, \sigma_{w}\right)$ - случайная перестановка множества $V$ (случайная нумерация вершин гиперграфа $H$ ), имеющая равномерное распределение на группе перестановок $S_{w}$, то есть для любой $\tilde{\sigma} \in S_{w}$

$$
\mathbf{P}(\sigma=\tilde{\sigma})=\frac{1}{w !} .
$$

Существование такого вероятностного пространства очевидно.

Пусть $e \in E-$ произвольное ребро $H$, а $i \in e-$ произвольная вершина этого ребра. Обозначим через $D_{e}^{i}$ и $\mathscr{D}_{i}$ следующие события:

$$
\begin{aligned}
& D_{e}^{i}=\left\{\sum_{s \in e} I\left\{\xi_{s}=\xi_{i}\right\}\left(1-I\left\{\sigma_{s}<\sigma_{i}, \eta_{s} \notin\left\{0, \xi_{s}\right\}\right\}\right)=n\right\}, \\
& \mathscr{D}_{i}=\bigcup_{e \in E: i \in e} D_{e}^{i} .
\end{aligned}
$$

Для каждого $i=1,2, \ldots, w$ положим

$$
\zeta_{i}=\xi_{i} I\left\{\overline{\mathscr{D}_{i}} \cup\left\{\eta_{i}=0\right\}\right\}+\eta_{i} I\left\{\mathscr{D}_{i} \cap\left\{\eta_{i} \neq 0\right\}\right\} .
$$


Несложно проверить, что случайные величины $\zeta_{i}$ принимают только значения из $A(i)$. Поэтому случайный вектор $\vec{\zeta}=\left(\zeta_{1}, \ldots, \zeta_{w}\right)$ можно интерпретировать как случайную раскраску множества $V$ (вершине $i$ сопоставляется цвет $\zeta_{i}$ ), соответствующую предписанию $A$. Обозначим через F्F событие, заключающееся в том, что случайная раскраска $\vec{\zeta}$ не является правильной раскраской гиперграфа $H$, то есть

$$
\mathscr{F}=\bigcup_{e \in E}\left\{\zeta_{s}=\zeta_{t} \text { для любых } s, t \in e\right\} .
$$

Имеет место следующее утверждение, которое из-за его важности мы представим в виде теоремы.

Теорема 4. Пусть $q=(r-1) p$. Тогда для произвольного n-равномерного гиперграфа $H$ с т ребрами, любого множества изветов $C \subset \mathbf{N}$ и любого вериинного предписания $A$ мощности $r$ выполнено неравенство

$$
\mathbf{P}(\mathscr{F}) \leqslant m r^{1-n}(1-q)^{n}+m^{2} r^{2-2 n} q .
$$

Доказательство теоремы 4 мы отнесем в следующий раздел, а сейчас продолжим доказательство теоремы 1.

Из построения случайной раскраски $\vec{\zeta}$ следует, что если для произвольного $n$-равномерного гиперграфа $H$, произвольного множества цветов $C \subset \mathbf{N}$ (а значит, и для множества цветов любой другой природы) и произвольного вершинного предписания $A$ мощности $r$ вероятность события F्F строго меньше единицы, то для $H$ существует правильная раскраска, соответствующая предписанию $A$. Значит, по определению, $H$ будет предписанно $r$-раскрашиваемым, что эквивалентно тому, что

$$
\chi_{p r}(H) \leqslant r .
$$

Теорема 4 дает универсальную оценку вероятности события $\mathscr{F}$. Таким образом, если для некоторых $m>0$ и $q \leqslant(r-1) / r$ выполняется неравенство

$$
m r^{1-n}(1-q)^{n}+m^{2} r^{2-2 n} q<1,
$$

то любой $n$-равномерный гиперграф, имеющий $m$ ребер, будет предписанно $r$-раскрашиваемым, и следовательно,

$$
m_{p r}(n, r)>m \text {. }
$$

Пусть

$$
m \leqslant c\left(\frac{n}{\ln n}\right)^{1 / 2} r^{n-1},
$$

где $c$ - любое положительное число, меньшее $\sqrt{3}-1$. Положим

$$
q=\frac{\ln (n / \ln n)}{2 n} .
$$

Тогда

$$
\begin{aligned}
m r^{1-n}(1-q)^{n}+m^{2} r^{2-2 n} q & \leqslant m r^{1-n} e^{-q n}+m^{2} r^{2-2 n} \frac{\ln n}{2 n} \\
& \leqslant c+\frac{c^{2}}{2}<1 .
\end{aligned}
$$


Следовательно, при таком подборе параметров выполнено условие (18), и значит, для любого $c<\sqrt{3}-1$ справедлива оценка

$$
m_{p r}(n, r) \geqslant c\left(\frac{n}{\ln n}\right)^{1 / 2} r^{n-1}
$$

Но если данное неравенство верно для всех $c<\sqrt{3}-1$, то оно верно и для $c=\sqrt{3}-1$.

Теорема 1 доказана.

\section{5. Доказательство теоремы 4}

Для удобства введем обозначения для некоторых событий. Пусть $e-$ ребро гиперграфа $H$, тогда положим

$$
\begin{aligned}
& A_{e}=\left\{\xi_{s}=\xi_{t}=\zeta_{s}=\zeta_{t} \text { для любых } s, t \in e\right\}, \\
& C_{e}=\left\{\zeta_{s}=\zeta_{t} \text { для любых } s, t \in e\right\} \cap\left(\bigcup_{s \in e}\left\{\xi_{s} \neq \zeta_{s}\right\}\right) .
\end{aligned}
$$

Для доказательства теоремы 4 нам понадобятся четыре леммы.

\section{1. Вспомогательные леммы}

Лемма 1. Пусть $е \in E-$ произвольное ребро гиперграфа, $\tilde{\sigma}-$ произвольная перестановка из $S_{w}$. Тогда следуюшие события совпадают:

$$
A_{1}=A_{e} \cap\{\sigma=\tilde{\sigma}\}, \quad A_{2}=\left\{\xi_{s}=\xi_{t}, \eta_{s} \in\left\{0, \xi_{s}\right\} \text { для любых } s, t \in e, \sigma=\tilde{\sigma}\right\} .
$$

Доказательство. Для любого $s$ из условия $\eta_{s} \in\left\{0, \xi_{s}\right\}$ следует, что $\zeta_{s}=\xi_{s}$, поэтому $A_{2} \subset A_{1}$. Докажем обратное включение.

Предположим, что $A_{1}$ выполнено, но в $e$ есть вершины, для которых $\eta_{s} \notin\left\{0, \xi_{s}\right\}$ (то есть $A_{2}$ неверно). Рассмотрим первую такую вершину $v$ по нумерации $\tilde{\sigma}$. Для нее, как нетрудно видеть, выполняется событие $\mathscr{D}_{v}$ и получается, что $\zeta_{v}=\eta_{v} \neq \xi_{v}$. Данный факт противоречит событию $A_{1}$. Таким образом, если выполнено событие $A_{1}$, то для любого $s \in e$ величина $\eta_{s} \in\left\{0, \xi_{s}\right\}$, то есть $A_{1} \subset A_{2}$. Лемма 1 доказана.

Лемма 2. Пусть $е \in E-$ произвольное ребро гиперграфа, $\tilde{\sigma}-$ произвольная перестановка из $S_{w}$. Тогда имеет место соотношение

$$
C_{e} \cap\{\sigma=\tilde{\sigma}\} \subset \quad \bigcup_{e f}(\tilde{\sigma}),
$$

где событие $B_{e f}(\tilde{\sigma})$ определяется равенством

$$
B_{e f}(\tilde{\sigma})=\left\{\zeta_{s}=\zeta_{t} \text { для любых } s, t \in e, \xi_{v} \neq \zeta_{v}=\eta_{v}, \bigcap_{s \in e} \overline{\left\{\sigma_{s}>\sigma_{v}, \xi_{s} \neq \zeta_{s}\right\}}, \sigma=\tilde{\sigma}\right\} \cap D_{f}^{v},
$$

здесь $v=f \cap e$. 
Доказательство. Событие $C_{e}$ означает, что для некоторых вершин $s \in e$ выполняется неравенство $\xi_{s} \neq \zeta_{s}$. Пусть $v-$ последняя по нумерации $\tilde{\sigma}$ вершина ребра $e$ с таким условием. Тогда для всех вершин $s \in e$ выполнено событие $\overline{\left\{\tilde{\sigma}_{s}>\tilde{\sigma}_{v}, \xi_{s} \neq \zeta_{s}\right\}}$.

Поскольку $\xi_{v} \neq \zeta_{v}$, во-первых, $\eta_{v}=\zeta_{v}$, а, во-вторых, должно выполняться событие $\mathscr{D}_{v}$, которое,в свою очередь, влечет выполнение одного из событий $D_{f}^{v}$, где $f$ - некоторое ребро, содержащее вершину $v$. Остается показать, что $|f \cap e|=1$.

Предположим, что, помимо $v$, найдется еще одна вершина $u \in f \cap e$. Тогда из события $D_{f}^{v}$ следует, что $\xi_{u}=\xi_{v}$, а из события $C_{e}$ вытекает равенство $\zeta_{u}=\zeta_{v}$. Значит, $\zeta_{u}=\eta_{u}=\eta_{v}$. В силу того, что $v$ - последняя по нумерации $\tilde{\sigma}$ вершина ребра $e$ с условием $\xi_{v} \neq \zeta_{v}$, получается, что $\tilde{\sigma}_{u}<\tilde{\sigma}_{v}$. Рассмотрим сумму из определения события $D_{f}^{v}$ (см. (15))

$$
D_{f}^{v} \cap\{\sigma=\tilde{\sigma}\}=\left\{\sum_{s \in f} I\left\{\xi_{s}=\xi_{v}\right\}\left(1-I\left\{\tilde{\sigma}_{s}<\tilde{\sigma}_{v}, \eta_{s} \notin\left\{0, \xi_{v}\right\}\right\}\right)=n\right\} .
$$

Слагаемое $s=u$, очевидно, дает нулевой вклад в сумму. Таким образом, общая сумма не может быть равна $n$, то есть не выполняется событие $D_{f}^{v}$. Мы получили противоречие. Следовательно, мощность пересечения ребер $е$ и $f$ равна единице. Лемма 2 доказана.

Лемма 3. Пусть е u $f$-два ребра гиперграфа $H$, причем $|f \cap e|=1, u v-$ вершина из пересечения этих ребер. Пусть перестановка б множества $V$ такова, что

$$
\left|\left\{s \in f: \tilde{\sigma}_{s}<\tilde{\sigma}_{v}\right\}\right|=z,
$$

$a\left|\left\{s \in e: \tilde{\sigma}_{s}<\tilde{\sigma}_{v}\right\}\right|=d$. Тогда имеет место следующая оиенка вероятности события $B_{e f}(\tilde{\sigma}):$

$$
\mathbf{P}\left(B_{e f}(\tilde{\sigma})\right) \leqslant r^{2-2 n} q(1-q)^{z}(1+q)^{d} \mathbf{P}(\sigma=\tilde{\sigma}) .
$$

Доказательство. Пусть выполнено событие $B_{e f}(\tilde{\sigma})$. Введем обозначения

$$
\begin{array}{ll}
f_{1}=\left\{s \in f: \tilde{\sigma}_{s}<\tilde{\sigma}_{v}\right\}, & f_{2}=\left\{s \in f: \tilde{\sigma}_{s}>\tilde{\sigma}_{v}\right\}, \\
e_{1}=\left\{s \in e: \tilde{\sigma}_{s}<\tilde{\sigma}_{v}\right\}, & e_{2}=\left\{s \in e: \tilde{\sigma}_{s}>\tilde{\sigma}_{v}\right\} .
\end{array}
$$

Ввиду события $D_{f}^{v}$, для всех вершин $s$ ребра $f$ выполнено событие $\left\{\xi_{s}=\xi_{v}\right\}$. Кроме того, для всех вершин $s \in f_{1}$ величины $\eta_{s}$ должны принимать значения 0 или $\xi_{s}$. Из условия $\bigcap_{s \in e} \overline{\left\{\tilde{\sigma}_{s}>\tilde{\sigma}_{v}, \xi_{s} \neq \zeta_{s}\right\}}$ получаем, что $\xi_{s}=\zeta_{s}$ для всех $s \in e_{2}$. Таким образом,

$$
\begin{aligned}
B_{e f}(\tilde{\sigma}) \subset & \left\{\sum_{s \in f_{1}} I\left\{\xi_{s}=\xi_{v}, \eta_{s} \in\left\{0, \xi_{s}\right\}\right\}=z, \sum_{s \in f_{2}} I\left\{\xi_{s}=\xi_{v}\right\}=n-z-1,\right. \\
& \left.\eta_{v} \notin\left\{\xi_{v}, 0\right\}, \sum_{s \in e_{1}} I\left\{\zeta_{s}=\eta_{v}\right\}=d, \sum_{s \in e_{2}} I\left\{\xi_{s}=\eta_{v}\right\}=n-d-1, \sigma=\tilde{\sigma}\right\} .
\end{aligned}
$$


Введем обозначение

$$
\begin{aligned}
& B_{e f}=\left\{\bigcap_{s \in f \backslash\{v\}}\left\{\left\{\xi_{s}=\xi_{v}, \eta_{s} \in\left\{0, \xi_{s}\right\}, \sigma_{s}<\sigma_{v}\right\} \sqcup\left\{\xi_{s}=\xi_{v}, \sigma_{s}>\sigma_{v}\right\}\right\} \cap\left\{\eta_{v} \notin\left\{\xi_{v}, 0\right\}\right\}\right. \\
& \cap \bigcap_{s \in e \backslash\{v\}}\left\{\left\{\xi_{s}=\eta_{v}, \sigma_{s}<\sigma_{v}\right\} \sqcup\left\{\xi_{s} \neq \eta_{v}, \eta_{s}=\eta_{v}, \sigma_{s}<\sigma_{v}\right\} \sqcup\left\{\xi_{s}=\eta_{v}, \sigma_{s}>\sigma_{v}\right\}\right\} .
\end{aligned}
$$

В силу (23) и того факта, что

$$
I\left\{\zeta_{s}=\eta_{v}\right\} \leqslant I\left\{\left\{\xi_{s}=\eta_{v}\right\} \sqcup\left\{\xi_{s} \neq \eta_{v}, \eta_{s}=\eta_{v}\right\}\right\},
$$

получаем соотношение

$$
B_{e f}(\tilde{\sigma}) \subset B_{e f} \cap\{\sigma=\tilde{\sigma}\} .
$$

Пусть $u, y \in A(v), u \neq y$; введем обозначения

$$
\begin{array}{ll}
B_{1}(u, y)=\sum_{s \in f_{1}} I\left\{\xi_{s}=y, \eta_{s} \in\{0, y\}\right\}, & B_{2}(u, y)=\sum_{s \in f_{2}} I\left\{\xi_{s}=y\right\}, \\
B_{3}(u, y)=\sum_{s \in e_{2}} I\left\{\xi_{s}=u\right\}, & B_{4}(u, y)=\sum_{s \in e_{1}} I\left\{\left\{\xi_{s}=u\right\} \sqcup\left\{\xi_{s} \neq u, \eta_{s}=u\right\}\right\} .
\end{array}
$$

Случайные величины $B_{i}(u, y), i=1, \ldots, 4$, при фиксированных $u, y$ независимы в совокупности. Из соотношений (24) и (25) получаем оценку вероятности события $B_{e f}(\tilde{\sigma})$ :

$$
\begin{aligned}
\mathbf{P}\left(B_{e f}(\tilde{\sigma})\right) & \leqslant \mathbf{P}\left(B_{e f} \cap\{\sigma=\tilde{\sigma}\}\right) \\
& \leqslant \sum_{u, y \in A(v), u \neq y} \mathbf{P}\left(B_{1}(u, y)=z, B_{2}(u, y)=n-z-1, B_{3}(u, y)=n-d-1\right. \\
& \leqslant r(r-1)\left(\frac{1-q}{r}\right)^{z}\left(\frac{1}{r}\right)^{n-z-1}\left(\frac{1}{r}\right)^{n-d-1}\left(\frac{1+q}{r}\right)^{d} \frac{p}{r} \mathbf{P}(\sigma=\tilde{\sigma}) \\
& =r^{2-2 n} q(1-q)^{z}(1+q)^{d} \mathbf{P}(\sigma=\tilde{\sigma} .
\end{aligned}
$$

Неравенство (17) обосновано. Лемма 3 доказана.

Лемма 4. Пусть е $u f-$ два ребра гиперграфа $H$, причем $|f \cap e|=1$. Обозначим через $v$ вершину из пересечения этих ребер. Рассмотрим случайные величины

$$
z=z(\sigma)=\left|\left\{s \in f: \sigma_{s}<\sigma_{v}\right\}\right|, \quad d=d(\sigma)=\left|\left\{s \in e: \sigma_{s}<\sigma_{v}\right\}\right| .
$$

Тогда для любого $q \in(0,1)$ имеет место неравенство

$$
\mathbf{M}(1-q)^{z}(1+q)^{d} \leqslant 1 .
$$


Доказательство. Несложно понять, что

$$
\begin{aligned}
\mathbf{M}(1-q)^{z}(1+q)^{d} & =\sum_{\tilde{\sigma} \in S_{w}}(1-q)^{z(\tilde{\sigma})}(1+q)^{d(\tilde{\sigma})} \mathbf{P}(\sigma=\tilde{\sigma}) \\
& =\sum_{i=0}^{n-1} \sum_{k=0}^{n-1}(1+q)^{i}(1-q)^{k}\left(\begin{array}{c}
n-1 \\
i
\end{array}\right)\left(\begin{array}{c}
n-1 \\
k
\end{array}\right) \frac{(i+k) !(2 n-2-i-k) !}{(2 n-1) !} \\
& =M(n-1, q) .
\end{aligned}
$$

Покажем, что имеет место соотношение $M(n, q) \leqslant M(n-1, q) \leqslant \ldots \leqslant M(0, q)=1$. Действительно, из тождества для биномиальных коэффициентов

$$
\left(\begin{array}{l}
n \\
i
\end{array}\right)=\left(\begin{array}{c}
n-1 \\
i
\end{array}\right)+\left(\begin{array}{l}
n-1 \\
i-1
\end{array}\right)
$$

вытекает следующее равенство

$$
\begin{aligned}
M(n, q)= & \sum_{i=0}^{n-1} \sum_{k=0}^{n-1}(1+q)^{i}(1-q)^{k}\left(\begin{array}{c}
n-1 \\
i
\end{array}\right)\left(\begin{array}{c}
n-1 \\
k
\end{array}\right) \frac{(i+k) !(2 n-i-k) !}{(2 n+1) !} \\
& +\sum_{i=1}^{n} \sum_{k=0}^{n-1}(1+q)^{i}(1-q)^{k}\left(\begin{array}{c}
n-1 \\
i-1
\end{array}\right)\left(\begin{array}{c}
n-1 \\
k
\end{array}\right) \frac{(i+k) !(2 n-i-k) !}{(2 n+1) !} \\
& +\sum_{i=0}^{n-1} \sum_{k=1}^{n}(1+q)^{i}(1-q)^{k}\left(\begin{array}{c}
n-1 \\
i
\end{array}\right)\left(\begin{array}{c}
n-1 \\
k-1
\end{array}\right) \frac{(i+k) !(2 n-i-k) !}{(2 n+1) !} \\
& +\sum_{i=1}^{n} \sum_{k=1}^{n}(1+q)^{i}(1-q)^{k}\left(\begin{array}{c}
n-1 \\
i-1
\end{array}\right)\left(\begin{array}{c}
n-1 \\
k-1
\end{array}\right) \frac{(i+k) !(2 n-i-k) !}{(2 n+1) !} .
\end{aligned}
$$

Изменив индексы суммирования в последних трех двойных суммах, получаем, что

$$
\begin{aligned}
M(n, q)= & \sum_{i=0}^{n-1} \sum_{k=0}^{n-1}(1+q)^{i}(1-q)^{k}\left(\begin{array}{c}
n-1 \\
i
\end{array}\right)\left(\begin{array}{c}
n-1 \\
k
\end{array}\right) \frac{(i+k) !(2 n-i-k) !}{(2 n+1) !} \\
& +\sum_{i=0}^{n-1} \sum_{k=0}^{n-1}(1+q)^{i+1}(1-q)^{k}\left(\begin{array}{c}
n-1 \\
i
\end{array}\right)\left(\begin{array}{c}
n-1 \\
k
\end{array}\right) \frac{(i+k+1) !(2 n-i-k-1) !}{(2 n+1) !} \\
& +\sum_{i=0}^{n-1} \sum_{k=0}^{n-1}(1+q)^{i}(1-q)^{k+1}\left(\begin{array}{c}
n-1 \\
i
\end{array}\right)\left(\begin{array}{c}
n-1 \\
k
\end{array}\right) \frac{(i+k+1) !(2 n-i-k-1) !}{(2 n+1) !} \\
& +\sum_{i=0}^{n-1} \sum_{k=0}^{n-1}(1+q)^{i+1}(1-q)^{k+1}\left(\begin{array}{c}
n-1 \\
i
\end{array}\right)\left(\begin{array}{c}
n-1 \\
k
\end{array}\right) \frac{(i+k+2) !(2 n-i-k-2) !}{(2 n+1) !} .
\end{aligned}
$$

Объединив снова все суммы в одну, получаем равенство

$$
M(n, q)=\sum_{i=0}^{n-1} \sum_{k=0}^{n-1}(1+q)^{i}(1-q)^{k}\left(\begin{array}{c}
n-1 \\
i
\end{array}\right)\left(\begin{array}{c}
n-1 \\
k
\end{array}\right) \frac{(i+k) !(2 n-2-i-k) !}{(2 n+1) !} \alpha,
$$

где

$\alpha=(2 n-i-k)(2 n-i-k-1)+2(i+k+1)(2 n-i-k-1)+(i+k+2)(i+k+1)\left(1-q^{2}\right)$. 
Величина $\alpha$, очевидно, не превосходит величины

$(2 n-i-k)(2 n-i-k-1)+2(i+k+1)(2 n-i-k-1)+(i+k+2)(i+k+1)=(2 n+1) 2 n$ при любых $i$ и $k$. Таким образом,

$$
\begin{aligned}
M(n, q) & \leqslant \sum_{i=0}^{n-1} \sum_{k=0}^{n-1}(1+q)^{i}(1-q)^{k}\left(\begin{array}{c}
n-1 \\
i
\end{array}\right)\left(\begin{array}{c}
n-1 \\
k
\end{array}\right) \frac{(i+k) !(2 n-2-i-k) !}{(2 n-1) !} \\
& =M(n-1, q) .
\end{aligned}
$$

Отсюда следует, что $M(n, q) \leqslant M(n-1, q) \leqslant \ldots \leqslant M(0, q)=1$. Из данной цепочки неравенств и соотношения (28) получаем искомую оценку (27). Лемма 4 доказана.

\section{2. Завершение доказательства теоремы 4}

В силу определений (19)

$$
\mathscr{F}=\bigcup_{e \in E}\left(A_{e} \cup C_{e}\right) .
$$

Из леммы 1 следует, что для любого ребра $e$ и его вершины $v$ выполняется неравенство

$$
\mathbf{P}\left(A_{e}\right) \leqslant \sum_{u \in A(v)} \mathbf{P}\left(\xi_{s}=u, \eta_{s} \in\{0, u\} \text { для любого } s \in e\right) \leqslant r^{1-n}(1-q)^{n} .
$$

Далее, из лемм 2, 3 и 4 получаем следующую оценку вероятности события $C_{e}$ :

$$
\begin{aligned}
\mathbf{P}\left(C_{e}\right) & \leqslant \sum_{f \in E:|f \cap e|=1} \sum_{\tilde{\sigma} \in S_{w}} \mathbf{P}\left(B_{e f}(\tilde{\sigma})\right) \\
& \leqslant \sum_{f \in E:|f \cap e|=1} \sum_{\tilde{\sigma} \in S_{w}} r^{2-2 n} q(1-q)^{z(\tilde{\sigma})}(1+q)^{d(\tilde{\sigma})} \mathbf{P}(\sigma=\tilde{\sigma}) \\
& =r^{2-2 n} q \sum_{f \in E:|f \cap e|=1} \mathbf{M}(1-q)^{z(\sigma)}(1+q)^{d(\sigma)} \leqslant r^{2-2 n} q \sum_{f \in E:|f \cap e|=1} 1 \leqslant m r^{2-2 n} q .
\end{aligned}
$$

Объединим полученные результаты. Из соотношений (29), (30) и (31) вытекает итоговая оценка вероятности события $\mathscr{F}$ :

$$
\begin{aligned}
\mathbf{P}(\mathscr{F}) & \leqslant \sum_{e \in E}\left(\mathbf{P}\left(C_{e}\right)+\mathbf{P}\left(A_{e}\right)\right) \leqslant \sum_{e \in E}\left(r^{1-n}(1-q)^{n}+m r^{2-2 n} q\right) \\
& =m r^{1-n}(1-q)^{n}+m^{2} r^{2-2 n} q .
\end{aligned}
$$

Искомое неравенство (17) обосновано. Теорема 4 доказана.

\section{6. Доказательство теоремы 2}

Для доказательства нам понадобится классическое утверждение, носящее название локальной леммы. Впервые оно было доказано в работе П. Эрдеша и Л. Ловаса [15]. Мы сформулируем данную теорему в специальном случае, удобном для дальнейших рассуждений. 
Теорема 5. Пусть на некотором вероятностном пространстве заданы события $Q_{1}, \ldots, Q_{N}$. Пусть $S_{1}, \ldots, S_{N}-$ подмножества множества $\mathscr{R}_{N}=\{1, \ldots, N\}$ такие, что для любого $i=1, \ldots, N$ событие $Q_{i}$ не зависит от алгебры, порожденной событиями $\left\{Q_{j}, j \in \mathscr{R}_{N} \backslash S_{i}\right\}$. Если для каждого $i=1, \ldots, N$ выполнены неравенства

$$
\begin{array}{r}
\mathbf{P}\left(Q_{i}\right)<1 / 2, \\
\sum_{j \in S_{i}} \mathbf{P}\left(Q_{j}\right) \leqslant 1 / 4,
\end{array}
$$

$m o \mathbf{P}\left(\bigcap_{j=1}^{N} \bar{Q}_{j}\right)>0$.

Доказательство локальной леммы можно найти в книге [18]. Отметим также, что если в условии теоремы каждое $j \in \mathscr{R}_{N}$ принадлежит хотя бы одному из множеств $S_{i}$, $i=1, \ldots, N$, то неравенство (32a) следует из (32b), поэтому для применения теоремы достаточно проверить только (32b).

Итак, пусть задан произвольный $n$-равномерный гиперграф $H=(V, E)$, удовлетворяющий условию теоремы 2. Пусть также задано конечное множество цветов $C$ и вершинное предписание $A$ мощности $r$, сопоставляющее каждой вершине $v \in V$ множество $A(v) \subset C$. Как и раньше, без ограничения общности можно считать, что $V=\{1,2,3, \ldots, w\}$ для некоторого натурального числа $w$, а $C \subset \mathbf{N}$. Построим случайную раскраску $\left\{\zeta_{s}\right\}_{s \in V}$ для $H$ точно так же, как это было сделано в доказательстве теоремы 1. Мы хотим показать, что $\chi_{p r}(H) \leqslant r$. Для этого достаточно доказать, что случайная раскраска $\left\{\zeta_{s}\right\}_{s \in V}$ будет (при некотором выборе параметра $p$ ) правильной раскраской $H \mathrm{c}$ положительной вероятностью.

Рассмотрим следующие наборы событий: $\mathscr{A}=\left\{A_{e}: e \in E\right\}$ (см. определение события $A_{e}$ в (19)), $\mathscr{B}=\left\{B_{e f}: e, f \in E,|e \cap f|=1\right\}$ (см. (24)). Из соотношений (16), (21), (25), (29) следует, что

$$
\left\{\text { раскраска }\left\{\zeta_{s}\right\}_{s \in V} \text { является правильной }\right\} \supset \bigcap_{Q \in \Re \cup \mathscr{B}} \bar{Q} .
$$

Остается показать, что вероятность последнего события строго больше нуля при некотором выборе параметра $p$. Его мы возьмем равным $p=\ln (n / \ln n) /(2(r-1) n)$. Ясно, что такой выбор $p$ корректен, то есть выполнено неравенство $r p \leqslant 1$.

Определим для каждого $Q \in \mathscr{A} \cup \mathscr{B}$ такое подмножество событий $S(Q) \subset \mathscr{A} \cup \mathscr{B}$, что $Q$ не зависит от алгебры, порожденной $\mathscr{A} \cup \mathscr{B} \backslash S(Q)$, и выполнено условие (32a)-(32b), которое в наших обозначениях выглядит так: для каждого $Q \in \mathscr{A} \cup \mathscr{B}$

$$
\begin{aligned}
\mathbf{P}(Q) & <1 / 2, \\
\sum_{Q_{0} \in S(Q)} \mathbf{P}\left(Q_{0}\right) & \leqslant 1 / 4 .
\end{aligned}
$$

Рассмотрим несколько случаев.

Первый случай. Пусть сначала $Q \in \mathcal{A}$, то есть $Q=A_{e}$ для некоторого $e \in E$. Из равенства (20) следует, что событие $A_{e}$ лежит в алгебре, порожденной случайными величинами $\left\{\xi_{s}, \eta_{s}: s \in e\right\}$. Поэтому оно не зависит от всех событий $A_{f}$, если $f \cap e=\varnothing$, от всех событий $B_{g} f$ при условии $g \cap e=\varnothing$ и $f \cap e=\varnothing$, а также от алгебры, порожденной этими событиями, потому что данная алгебра лежит в алгебре, порожденной $\sigma$ и $\left\{\xi_{s}, \eta_{s}: s \in\right.$ $V \backslash e\}$. Таким образом, в качестве $S\left(A_{e}\right)$ достаточно взять подмножество $\mathcal{A}$, состоящее из событий $A_{f}, f \cap e \neq \varnothing$, и подмножество $\mathscr{B}$, состоящее из событий $B_{g}$ с условием, что 
либо $f \cap e \neq \varnothing$, либо $g \cap e \neq \varnothing$. По условию теоремы 2 событий первого вида не более $d$, а второго не более, чем $2 d^{2}$. Из неравенств (26), (27) и (30) получаем оценку суммы вероятностей

$$
\begin{aligned}
\sum_{Q \in S\left(A_{e}\right)} \mathbf{P}(Q) & \leqslant \sum_{f:|f \cap e| \geqslant 1} \mathbf{P}\left(A_{f}\right)+\sum_{f:|f \cap e| \geqslant 1} \sum_{g:|f \cap g|=1}\left(\mathbf{P}\left(B_{f g}\right)+\mathbf{P}\left(B_{g f}\right)\right) \\
& \leqslant d r^{1-n}(1-q)^{n}+2 d^{2} r^{2-2 n} q \leqslant \frac{\sqrt{6}-2}{4}+\left(\frac{\sqrt{6}-2}{4}\right)^{2} \leqslant \frac{1}{4} .
\end{aligned}
$$

Предпоследнее неравенство в этой цепочке выполняется в силу выбора параметра $p=q /(r-1)$, а также выражения (9) для величины $d$. Таким образом, в случае, когда $Q=A_{e}$, мы доказали выполнение условия (33b). Условие же (33a) очевидным образом выполняется для любого $Q \in \mathscr{A} \cup \mathscr{B}$.

Второй случай. Остается разобрать случай, когда $Q \in \mathscr{B}$, то есть $Q=B_{e f}$. Из определения события $B_{e f}$ (см. (24)) следует, что оно лежит в алгебре, порожденной случайными величинами $\left\{\xi_{s}, \eta_{s}: s \in e \cup f\right\}$ и набором $\left\{I\left\{\sigma_{s}<\sigma_{t}\right\}: s, t \in e \cup f\right\}$. Отсюда получается, что данное событие не зависит от всех событий $A_{g}$, если $g \cap(e \cup f)=\varnothing$, от всех событий $B_{g h}$ при условии $(g \cup h) \cap(e \cap f)=\varnothing$, а также от алгебры, порожденной этими событиями, потому что данная алгебра лежит в алгебре, порожденной случайными величинами $\left\{\xi_{s}, \eta_{s}: s \in V \backslash(e \cup f)\right\}$ и $\left\{I\left\{\sigma_{s}<\sigma_{t}\right\}: s, t \in V \backslash(e \cup f)\right\}$. Таким образом, в качестве $S\left(B_{e f}\right)$ достаточно взять подмножество $\mathcal{A}$, состоящее из событий $A_{g}, g \cap(e \cup f) \neq \varnothing$, и подмножество $\mathscr{B}$, состоящее из событий $B_{g h}$ с условием $(g \cup h) \cap(e \cup f) \neq \varnothing$. По условию теоремы событий первого типа не больше, чем $2 d$, второго - не больше, чем $4 d^{2}$. Как и в первом случае, из неравенств (26), (27) и (30) получаем оценку суммы вероятностей

$$
\begin{aligned}
\sum_{Q \in S\left(B_{e f}\right)} \mathbf{P}(Q) \leqslant & \sum_{g:|g \cap e| \geqslant 1} \mathbf{P}\left(A_{g}\right)+\sum_{g:|g \cap f| \geqslant 1} \mathbf{P}\left(A_{g}\right)+\sum_{g:|g \cap e| \geqslant 1} \sum_{h:|h \cap g|=1}\left(\mathbf{P}\left(B_{h g}\right)+\mathbf{P}\left(B_{g h}\right)\right) \\
& +\sum_{g:|g \cap f| \geqslant 1} \sum_{h:|h \cap g|=1}\left(\mathbf{P}\left(B_{h g}\right)+\mathbf{P}\left(B_{g h}\right)\right) \\
& \leqslant 2 d r^{1-n}(1-q)^{n}+4 d^{2} r^{2-2 n} q \leqslant \frac{\sqrt{6}-2}{2}+\frac{(\sqrt{6}-2)^{2}}{4}=\frac{1}{4} .
\end{aligned}
$$

Неравенство (33b) доказано и во втором случае.

Подведем итоги. Для системы событий $\mathscr{A} \cup \mathscr{B}$ выполнено условие (32a)-(32b) локальной леммы. Из нее следует, что с положительной вероятностью не случится ни одно из этих событий, поэтому для $H$ существует правильная раскраска, соответствующая предписанию $A$. Теорема 4 доказана.

\section{7. Доказательство теоремы 3}

Для доказательства нам понадобится несколько простых лемм, аналогичных утверждениям, с помощью которых Эрдешем и Ловасом была доказана оценка (11).

Пусть $H=(V, E)$ - произвольный простой $n$-равномерный гиперграф, для которого $\chi_{p r}(H)>r$. Покажем, что

$$
|E| \geqslant \frac{(\sqrt{6}-2)^{2}}{32} \frac{r^{2 n-4}}{n \ln n} .
$$


Положим величину $d(n, r)$ равной правой части равенства (9) из условия теоремы 2 , то есть

$$
d(n, r)=\frac{\sqrt{6}-2}{4}\left(\frac{n}{\ln n}\right)^{1 / 2} r^{n-1} .
$$

Лемма 5. Множество $E$ содержит ребро, пересекающее более $d(n, r)$ других ребер гиперграфа Н. В этом ребре есть вериина $v$ такая, что

$$
\operatorname{deg} v \geqslant \frac{d(n, r)}{n}+1
$$

Доказательство. Первое утверждение прямо следует из теоремы 2. Если бы такого ребра не было, то $\chi_{p r}(H)$ не превосходило бы $r$, что противоречит выбору гиперграфа $H$. Пусть $e \in E-$ искомое ребро. Далее, в силу того, что гиперграф $H$ простой,

$$
\sum_{w \in e} \operatorname{deg} w=|\{f \in E: f \cap e \neq \varnothing, f \neq e\}|+n \geqslant d(n, r)+n .
$$

В ребре $e$ содержится ровно $n$ вершин, поэтому в нем найдется вершина $v$ такая, что $\operatorname{deg} v \geqslant d(n, r) / n+1$. Лемма 5 доказана.

Лемма 6. Множество $H$ содержит не менее $d(n-1, r) /(n-1)+1$ вериин, степень каждой из которых не меньше, чем $d(n-1, r) /(n-1)+1$.

Доказательство. Для каждого ребра $e \in E$ через $v(e)$ обозначим вершину ребра $e$, имеющую максимальную степень (если таких вершин несколько, то возьмем любую из них). Рассмотрим гиперграф $H_{1}=\left(V, E_{1}\right)$, где $E_{1}=\{e \backslash\{v(e)\}: e \in E\}$. Это $(n-1)$-равномерный гиперграф, причем $\chi_{p r}\left(H_{1}\right)>r$. Если бы $H_{1}$ был предписанно $r$-раскрашиваемым, то таким же был бы и исходный гиперграф $H$. Из леммы 5 следует, что у $H_{1}$ есть вершина $w$, степень которой не меньше, чем $d(n-1, r) /(n-1)+1$. Пусть $f_{1}, \ldots, f_{\operatorname{deg}} w-$ ребра $H_{1}$, содержащие $w$, а $e_{1}, \ldots, e_{\operatorname{deg} w}-$ “восстановленные" по $f_{1}, \ldots, f_{\operatorname{deg}} w$ ребра гиперграфа $H$, то есть $f_{j}=e_{j} \backslash\left\{v\left(e_{j}\right)\right\}, j=1 \ldots, \operatorname{deg} w$. Тогда для каждого $j=1, \ldots, \operatorname{deg} w$ получаем, что $\operatorname{deg}_{H} v\left(e_{j}\right) \geqslant \operatorname{deg}_{H} w \geqslant \operatorname{deg} w \geqslant d(n-1, r) /(n-1)+1$.

Остается выяснить, почему вершины $v\left(e_{1}\right), \ldots, v\left(e_{\operatorname{deg} w}\right)$ различны. Если бы $v\left(e_{j}\right)=v\left(e_{k}\right)$, то ребра $e_{j}$ и $e_{k}$ имели бы две общие вершины (все эти ребра содержат $w)$, что противоречит тому, что $H$ - простой гиперграф. Лемма 6 доказана.

Лемма 7. Гиперграф $H$ содержит не менее

$$
\left\lceil\frac{d(n-1, r)}{n-1}+1\right\rceil+\left(\left\lceil\frac{d(n-1, r)}{n-1}+1\right\rceil-1\right)+\ldots+1=\left(\begin{array}{c}
\lceil d(n-1, r) /(n-1)\rceil+1 \\
2
\end{array}\right)
$$

ребер.

Доказательство. Воспользуемся леммой 6. Положим $m=\lceil d(n-1, r) /(n-1)\rceil+1$. В силу того, что число $d(n-1, r) /(n-1)$ не является целым, в $H$ существуют вершины $v_{1}, \ldots, v_{m}$, степени которых не меньше, чем $m$. Тогда $|E| \geqslant\left|E_{1}\right|+\left|E_{2}\right|+\ldots+\left|E_{m}\right|$, где $E_{j}=\left\{e \in E: v_{j} \in E, v_{k} \notin E, k=1, \ldots, j-1\right\}$. Ввиду того, что гиперграф $H$ простой, получаем, что $\left|E_{j}\right| \geqslant \operatorname{deg} v_{j}-j+1 \geqslant m-j+1$. Следовательно,

$$
|E| \geqslant \sum_{j=1}^{m}(m-j+1)=\left(\begin{array}{l}
m \\
2
\end{array}\right) \text {. }
$$

Лемма 7 доказана. 
Завершим доказательство теоремы 3. Из леммы 7 следует, что

$$
\begin{aligned}
|E| & \geqslant\left(\begin{array}{c}
\lceil d(n-1, r) /(n-1)\rceil+1 \\
2
\end{array}\right) \geqslant \frac{1}{2}\left(\frac{d(n-1, r)}{n-1}\right)^{2} \\
& =\frac{(\sqrt{6}-2)^{2}}{32} \frac{r^{2 n-4}}{(n-1) \ln (n-1)} \geqslant \frac{(\sqrt{6}-2)^{2}}{32} \frac{r^{2 n-4}}{n \ln n} .
\end{aligned}
$$

Неравенство (34) обосновано. Теорема 3 доказана.

\section{Список литературы}

1. Erdős P., Hajnal A., On a property of families of sets. Acta Math. Acad. Sci. Hung. (1961) 12, $87-123$.

2. Erdös P., Some old and new problems in various branches of combinatorics. In: Proc. 10th Southeastern Conf. on Combinatorics, Graph Theory and Computing. Utilitas Mathematica, Winnipeg, 1979, pp. 19-37.

3. Erdős P., On a combinatorial problem. I. Nordisk Mat. Tidskrift (1963) 11, 5-10.

4. Erdős P., On a combinatorial problem. II. Acta Math. Acad. Sci. Hung. (1964) 15, 445-447.

5. Kostochka A. V., Color-critical graphs and hypergraphs with few edges: a survey. Bolyai Soc. Math. Stud. (2006) 15, 175-198.

6. Schmidt W. M. Ein kombinatorisches Problem von P. Erdős and A. Hajnal. Acta Math. Acad. Sci. Hung. (1964) 15, 373-374.

7. Beck J., On a combinatorial problem of P. Erdős and L. Lovász. Discrete Math. (1977) 17, 127-131.

8. Beck J., On 3-chromatic hypergraphs. Discrete Math. (1978) 24, 127-137.

9. Spencer J. H., Coloring $n$-sets red and blue. Combinatorial Theory, Ser. A (1981) 30, 112-113.

10. Radhakrishnan J., Srinivasan A., Improved bounds and algorithms for hypergraph two-coloring. Random Structures and Algorithms (2000) 16, 4-32.

11. Alon N., Hypergraphs with high chromatic number. Graphs and Combinatorics (1985) 1, 387-389.

12. Kostochka A. V., Coloring uniform hypergraphs with few colors. Random Structures and Algorithms (2004) 24, 1-10.

13. Визинг В. Г., Раскраска вершин графа в предписанные цвета. В сб.: Методы дискретного анализа в теории кодов и схем, 29. Институт математики СО АН СССР, Новосибирск, 1976, c. $3-10$.

14. Erdős P., Rubin A. L., Taylor H., Choosability in graphs. In: Proc. West Coast Conference on Combinatorics, Graph Theory and Computing, Arcata/Calif., 26, 1980, pp. 125-157.

15. Erdős P., Lovász L., Problems and results on 3-chromatic hypergraphs and some related questions. Colloq. Math. Soc. Janos Bolyai (1975) 10, 609-627.

16. Szabo Z., An application of Lovasz local lemma-a new lower bound for the van der Waerden number. Random Structures and Algorithms (1990) 1, 343-360.

17. Grable B., Phelps K., Rödl V., The minimum independence number for designs. Combinatorica (1995) 15, 175-185.

18. Алон Н., Спенсер Дж., Вероятностный метод. Бином, Москва, 2007. 